\title{
The double degenerate system NLTT 11748 ^
}

\author{
A. Kawka ${ }^{1}$, S. Vennes ${ }^{1,2}$, and T. R. Vaccaro ${ }^{2,3}$ \\ 1 Astronomický ústav, Akademie věd České republiky, Fričova 298, 25165 Ondřejov, Czech Republic \\ e-mail: [kawka; vennes]@sunstel .asu.cas.cz \\ 2 Visiting Astronomer, Kitt Peak National Observatory, National Optical Astronomy Observatory, which is operated by the \\ Association of Universities for Research in Astronomy (AURA) under cooperative agreement with the National Science Foundation \\ 3 Department of Physics \& Astronomy, Francis Marion University, Box 100547, Florence, SC 29501, USA \\ e-mail: tvaccaro@fmarion.edu
}

Received 15 April 2010 / Accepted 18 May 2010

\section{ABSTRACT}

\begin{abstract}
We show that the extremely low-mass white dwarf NLTT $11748\left(0.17 M_{\odot}\right)$ is in a close binary with a fainter companion. We obtained a series of radial velocity measurements of the low-mass white dwarf using the $\mathrm{H} \alpha$ core and determined an orbital period of 5.64 h. The velocity semi-amplitude $\left(K=274.8 \mathrm{~km} \mathrm{~s}^{-1}\right)$ and orbital period imply that it is a degenerate star, and that the minimum mass for the companion is $0.75 M_{\odot}$ (assuming a mass of $0.167 M_{\odot}$ for the primary). Our analysis of Balmer line profiles shows that a $0.75 M_{\odot}$ white dwarf companion does not contribute more than $2 \%$ or $5 \%$ of the flux ( $V$-band) for helium- or hydrogen-rich surfaces, respectively. The kinematics of the system suggest that it belongs to the Galactic halo.
\end{abstract}

Key words. binaries: close - stars: individual: NLTT 11748 - white dwarfs

\section{Introduction}

The high proper-motion star NLTT 11748 was identified as an extremely low mass (ELM) white dwarf by Kawka \& Vennes (2009). The first ELM white dwarfs were discovered to be companions to neutron stars (e.g., van Kerkwijk et al. 1996; Bassa et al. 2003), but recent follow-up observations of ELM white dwarfs identified in colourimetric or proper motion surveys (e.g., SDSS, NLTT) often find them to be in a close binary with a white dwarf companion (e.g., SDSS J125733.63+542850.5, Badenes et al. 2009; Kulkarni \& van Kerkwijk 2010; Marsh et al. 2010). The companions of several other ELM white dwarfs found to be in close binary systems are yet to be formally identified, but they are likely to be more massive white dwarfs (see, Agüeros et al. 2009a,b).

The stellar and kinematical properties of NLTT 11748 (Kawka \& Vennes 2009) and of two similar objects, LP400-22 (Kilic et al. 2009; Vennes et al. 2009) and SDSS J1053+5200 (Kilic et al. 2010), also suggest that they are old halo stars. The constraints placed on their total ages help retrace the prior evolution of these systems (see Tauris \& Savonije 1999; Nelemans et al. 2001; Nelson et al. 2004).

The formation of ELM white dwarfs requires that the systems go through at least two phases of mass transfer, where the second mass transfer phase strips the less massive companion of its outer envelope before the helium ignition. This generic scenario appears valid whether the more massive component is a neutron star or a white dwarf (see for example Nelemans et al. 2001; Nelson et al. 2004). An example of a possible progenitor to an ELM white dwarf is the bright subluminous B ( $\mathrm{sdB}$ ) star, HD 188112 (Heber et al. 2003). The subdwarf star has a lower mass than average, 0.24 versus $\sim 0.5 M_{\odot}$ (Zhang et al. 2009),

* Based on observations at the European Organisation for Astronomical Research in the Southern Hemisphere, Chile under programme ID 84.D-0862. and orbits a degenerate companion with a mass $\gtrsim 0.73 M_{\odot}$ every $14.6 \mathrm{~h}$. The subdwarf is a likely progenitor of a helium white dwarf similar to NLTT 11748. On the other hand, a possible progenitor to an ELM white dwarf with, this time, a neutron star companion is SDSS J102347.6+003841 (Wang et al. 2009). This is a close binary comprising a G-type star and a $1.69 \mathrm{~ms}$ pulsar (Archibald et al. 2009) with an orbital period of $4.75 \mathrm{~h}$. Although the spectral type of the visible component implies a mass of $\approx 1 M_{\odot}$, the mass ratio of the system dictates a much lower mass of $\sim 0.2 M_{\odot}$ suggesting that the star is significantly evolved with a helium-enriched core. This system probably represents a first link between low-mass X-ray binaries and ELM plus neutron star binaries (Archibald et al. 2009).

We report new observations that help clarify the nature of the ELM white dwarf NLTT 11748. We obtained two sets of intermediate resolution spectra that show that NLTT 11748 is in a close binary system (Sect. 2). Our analysis of the spectroscopic data and a first determination of the binary parameters are presented in Sect. 3. We discuss our results in Sect. 4 and summarise in Sect. 5.

\section{Observations}

We observed NLTT 11748 with the FORS2 spectrograph attached to the UT1 - Antu at Paranal on UT 2009 October 22 and 23 . We used the $1200 \mathrm{R}$ grism combined with a slit width of $1^{\prime \prime}$, which provided a resolution of $3.0 \AA$. The spectra were obtained as part of a programme aimed at detecting and measuring magnetic fields in hydrogen-rich white dwarf stars. The observation sequence consists of two consecutive exposures with the Wollaston prism rotated by 90 degrees between them. In principle, a magnetic field may cause an apparent velocity offset in pairs of exposures, but our analysis of the radial velocity measurements excludes the effect of a magnetic field (Sect. 3.1). 

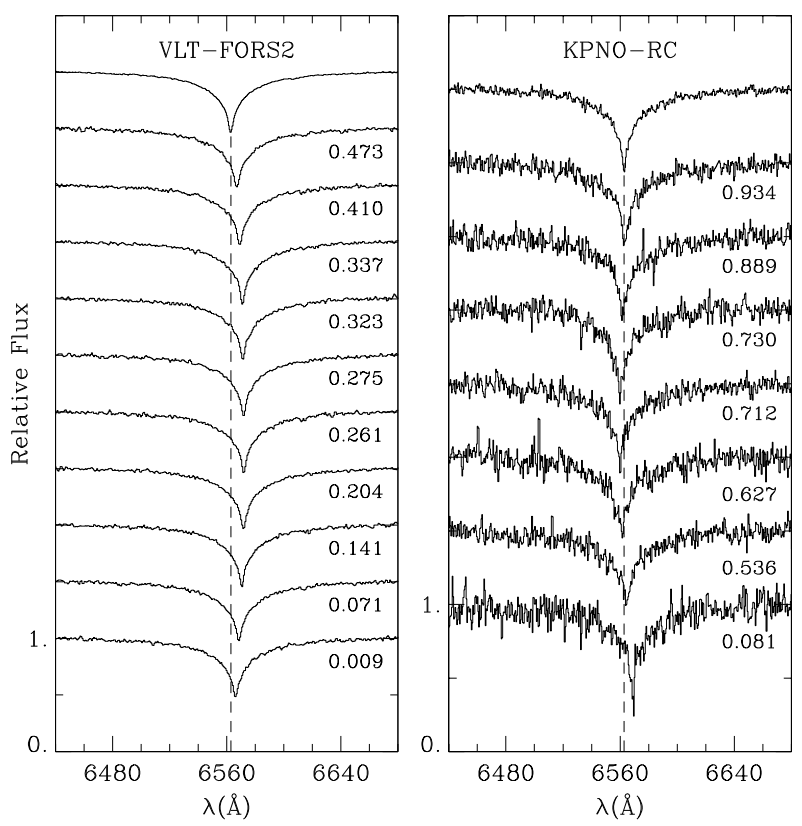

Fig. 1. Spectra obtained with VLT FORS2 (left) and the KPNO R-C spectrograph (right). The spectra are labeled with the orbital phase (Sect. 3.1) and the top spectra are the co-added phase-corrected spectrum for each spectrograph.

We obtained additional observations with the R-C spectrograph attached to the $4 \mathrm{~m}$ telescope at the Kitt Peak National Observatory (KPNO) on UT 2010 March 4 to 6 . We used the T2KB detector and the KPC-24 grating (860 lines $/ \mathrm{mm}$ ) in second order centred on $\mathrm{H} \alpha$. We employed the GG495 filter to block out contamination from the first order. We set the slit width to $1.5^{\prime \prime}$, which provided a resolution of $0.8 \AA$ (full-width half-maximum)

All spectra were wavelength-calibrated using comparison arc (HeNeAr) exposures. The stability of the wavelength scale was verified through OI sky lines, which remained stable with a standard deviation of $3 \mathrm{~km} \mathrm{~s}^{-1}$. All data were reduced with standard procedures within IRAF. Figure 1 and Table 1 show our spectra and the observation log.

\section{Analysis}

\subsection{Binary parameters}

We used the $\mathrm{H} \alpha$ line centre to measure the radial velocity of NLTT 11748. We fitted a Gaussian profile to the deep, narrow core. Repeated measurements varying the fitting window from 2 to $5 \AA$ from the line centre were averaged, leaving residuals of 3 to $10 \mathrm{~km} \mathrm{~s}^{-1}$ depending on the signal-to-noise ratio of the spectrum. The velocities were then corrected to the solar system barycentre. We verified the procedure by cross-correlating each spectrum with a template built by co-adding all spectra in the rest frame (see Fig. 1) and comparing the resulting velocities to the Gaussian fits. With a window from 6500 to $6620 \AA$, we found that the two methods differ by $\Delta v=1.4 \pm 3.5 \mathrm{~km} \mathrm{~s}^{-1}$ for the higher quality FORS2 spectra, and $\Delta v=3.2 \pm 12.0 \mathrm{~km} \mathrm{~s}^{-1}$ for the R-C spectra. Accordingly, the FORS2 and R-C spectra are weighted 4:1 in the orbital analysis. Table 2 lists the barycentric mid-exposure times and velocities.

We determined the orbital parameters by fitting sinusoidal functions to the radial velocities, where the period $(P)$, mean systemic velocity $(\gamma)$, the velocity semi-amplitude $(K)$ and the
Table 1. Observation log.

\begin{tabular}{lccc}
\hline \hline Start UT date and time & $t_{\exp }(s)$ & Start UT date and time & $t_{\text {exp }}(s)$ \\
\hline \multicolumn{5}{c}{ ESO } \\
2009 Oct. 22 04:51:00 & 1200 & 2009 Oct. 22 06:42:14 & 1200 \\
2009 Oct. 22 05:12:09 & 1200 & 2009 Oct. 23 04:50:11 & 1200 \\
2009 Oct. 22 05:35:52 & 1200 & 2009 Oct. 23 05:11:20 & 1200 \\
2009 Oct. 22 05:57:00 & 1200 & 2009 Oct. 23 05:40:44 & 1200 \\
2009 Oct. 22 06:21:05 & 1200 & 2009 Oct. 23 06:01:52 & 1200 \\
\hline \multicolumn{5}{c}{ KPNO } \\
2010 Mar. 04 03:19:37 & 1800 & 2010 Mar. 05 03:53:24 & 1800 \\
2010 Mar. 04 03:50:25 & 1800 & 2010 Mar. 05 04:58:15 & 1800 \\
2010 Mar. 04 04:25:32 & 1800 & 2010 Mar. 06 02:42:39 & 1800 \\
2010 Mar. 05 02:53:19 & 1800 & \\
\hline
\end{tabular}

Table 2. Radial velocity measurements of NLTT 11748.

\begin{tabular}{cccc}
\hline \hline $\begin{array}{c}\text { BJD } \\
(2455000+)\end{array}$ & $\begin{array}{c}v_{\text {bary }} \\
\left(\mathrm{km} \mathrm{s}^{-1}\right)\end{array}$ & $\begin{array}{c}\text { BJD } \\
(2455000+)\end{array}$ & $\begin{array}{c}v_{\text {bary }} \\
\left(\mathrm{km} \mathrm{s}^{-1}\right)\end{array}$ \\
\hline \multicolumn{4}{c}{ VLT/FORS2 } \\
126.71404 & 151.4 & 126.79129 & 369.5 \\
126.72873 & 259.2 & 127.71352 & 412.2 \\
126.74520 & 354.1 & 127.72821 & 380.7 \\
126.75988 & 403.4 & 127.74863 & 286.5 \\
126.77660 & 410.7 & 127.76330 & 189.2 \\
\hline \multicolumn{4}{c}{ KPNO/R-C } \\
259.64754 & 87.1 & 260.67090 & -38.4 \\
259.66893 & -64.6 & 260.71593 & 269.0 \\
259.69331 & -131.7 & 261.62168 & 23.5 \\
260.62918 & -131.1 & \\
\hline
\end{tabular}

initial epoch $\left(T_{0}\right)$ are varied. Figure 2 shows the periodogram and best-fit radial velocity curve. The significance values are drawn at $66 \%, 90 \%$ and $99 \%$. The average of the residuals is $\sim 3 \mathrm{~km} \mathrm{~s}^{-1}$, commensurate with our estimates of the wavelength scale accuracy. Although the FORS2 spectra were obtained with the Wollaston prism in place, the low residual value excludes a significant magnetic field. A more detailed spectropolarimetric analysis will be reported elsewhere.

The best-fit parameters are

$P=0.235061 \pm 0.000003 \mathrm{~d}$,

$T_{0}(\mathrm{BJD} \mathrm{UT})=2455126.712 \pm 0.003$,

where $T_{0}$ is the initial epoch of superior conjunction of the unseen companion. We also determined the systemic velocity

$\gamma_{\mathrm{sym}}=136.5 \pm 0.9 \mathrm{~km} \mathrm{~s}^{-1}$

by subtracting the gravitational redshift of the white dwarf $\left(v_{\mathrm{g}}=2.0 \pm 0.4 \mathrm{~km} \mathrm{~s}^{-1}\right)$ from the apparent systemic velocity of $\gamma=138.5 \pm 0.8 \mathrm{~km} \mathrm{~s}^{-1}$. The gravitational redshift of the white dwarf was calculated assuming the parameters derived in Kawka \& Vennes (2009). The observed velocity semi-amplitude is

$K=274.8 \pm 1.5 \mathrm{~km} \mathrm{~s}^{-1}$

which corresponds to a mass function for the unseen companion $f=0.5054 \pm 0.0083 M_{\odot}$.

Adopting a mass of $0.167 M_{\odot}$ for the white dwarf (Kawka \& Vennes 2009) and using our orbital parameters, we infer a minimum mass for the unseen companion of $0.75 M_{\odot}$. A mainsequence companion with a mass of $0.75 M_{\odot}$ would have an absolute magnitude of $M_{V} \sim 7$ (Henry \& McCarthy 1993), which would clearly outshine a white dwarf with $M_{V}=9.7$. 
A. Kawka et al.: The double degenerate system NLTT 11748
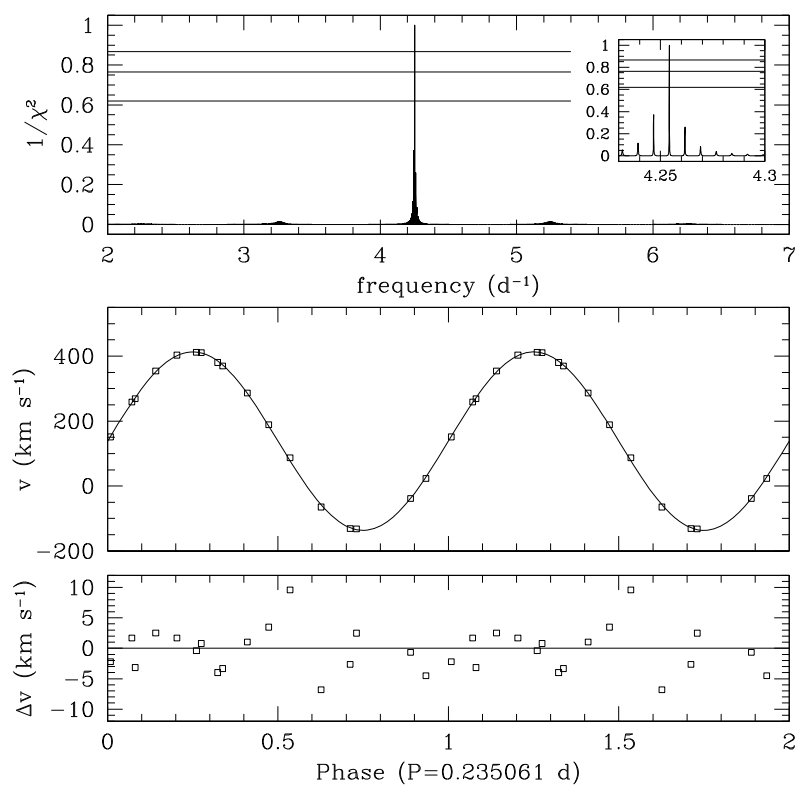

Fig. 2. (Top) Periodogram of radial velocity measurements showing, in inset, an enlarged segment near the best period. (Middle) Radial velocity measurements folded on the orbital period and compared to a sinusoidal fit (see text). (Bottom) Residuals of the sinusoidal fit.

We re-evaluated the kinematics of the system with the new systemic velocity to obtain $(U, V, W)=(-163,-252$, $-29) \mathrm{km} \mathrm{s}^{-1}$. These updated values clearly place NLTT 11748 in the halo. Two other ELM systems whose kinematics place them in the halo are LP400-22 (Vennes et al. 2009) and SDSS J1053+5200 (Kilic et al. 2010) with the remaining systems (without a neutron star) apparently belonging to the disc (Kilic et al. 2010).

\subsection{Constraints on a white dwarf companion}

Although Kawka \& Vennes (2009) achieved a satisfactory fit to the Balmer line profiles, their analysis did not consider a companion. We repeated that analysis using two composite model grids, DA+DC or DA+DA. We modelled the primary with a pure-hydrogen model grid (DA) and the secondary with either a pure-helium (DC) or a DA grid. First, we set the mass of the secondary to $0.75 M_{\odot}$. We fitted the Balmer line profiles (see Kawka \& Vennes 2009, for details of the NTT-EFOSC2 spectrum) varying three parameters: the temperature and surface gravity of the primary $\left(T_{\text {eff, }}, \log g_{\mathrm{p}}\right)$ and the effective temperature of the secondary $\left(T_{\mathrm{eff}, \mathrm{s}}\right)$. The total flux was obtained by combining the primary and secondary surface fluxes weighted by the emitting area: $R^{2} f_{\text {tot }}=R_{\mathrm{p}}^{2} f_{\mathrm{p}}+R_{\mathrm{s}}^{2} f_{\mathrm{s}}$, where $R$ is the effective radius of the composite star, and $R_{\mathrm{p}}$ and $R_{\mathrm{S}}$ are the primary and secondary radii computed using the evolutionary mass radius relations of Benvenuto \& Althaus (1999) and Serenelli et al. (2002). Note that the flux composition takes into account the calculated orbital phase of the observed spectrum and the corresponding offset in velocities between the two stars. The combined spectrum is then fitted to the observed spectrum with $\chi^{2}$ minimization techniques.

The best-fit parameters from the DA+DA grid are $T_{\text {eff,p }}=$ $8580 \pm 50 \mathrm{~K}(1 \sigma)$ and $\log g_{\mathrm{p}}=6.18 \pm 0.15$ for the primary and $T_{\text {eff,s }} \leq 9600 \mathrm{~K}$ for the secondary. The flux contribution from the secondary at $5500 \AA$ ( $V$-band) is $\leq 5 \%$ of the total flux. But if we used the DA+DC grid we obtained an upper limit of $T_{\text {eff,s }} \leq 7200 \mathrm{~K}$, with a flux contribution $\leq 2 \%$. Setting the

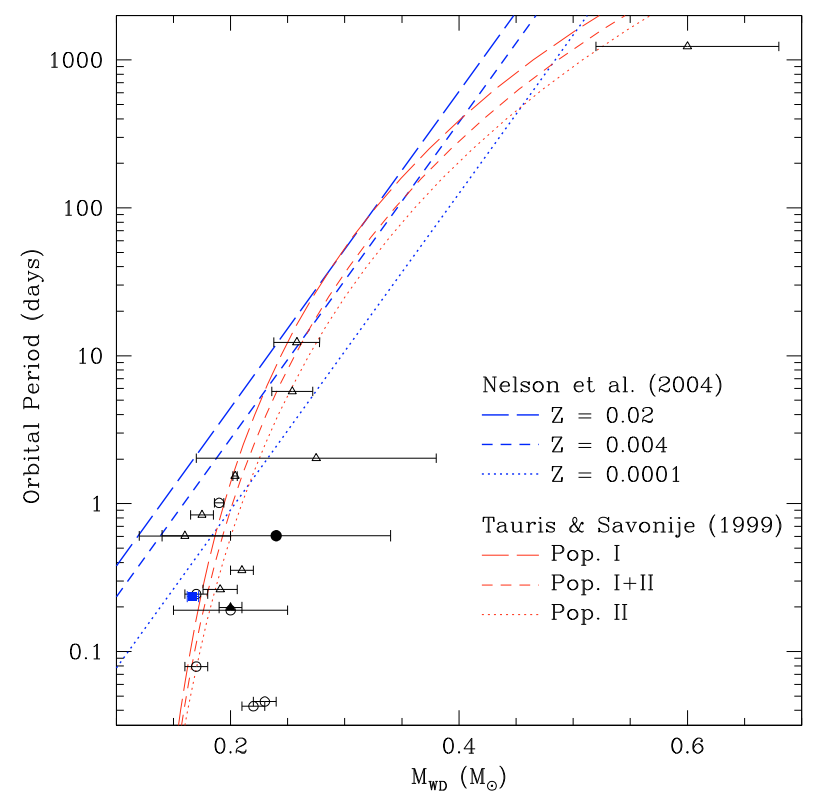

Fig. 3. Orbital period versus mass of the visible component in binary systems containing a white dwarf and a neutron star (open triangles) or another white dwarf (open circles, this includes systems where the unseen is unknown, but has a high probability of being a more massive white dwarf). NLTT 11748, HD 188112, and SDSS J1023+0038 are marked with a filled square, circle, and triangle, respectively. These values are compared to theoretical period-mass relations for NS+WD systems of Nelson et al. (2004) and Tauris \& Savonije (1999) drawn with thick and thin lines, respectively.

secondary mass to $1 M_{\odot}$ did not affect our solution for the primary, but allowed a higher secondary temperature $(+400 \mathrm{~K})$. In summary, the best-fit parameters are identical to the single star solution obtained by Kawka \& Vennes (2009), and we conclude that the secondary star is much fainter than the primary star.

\section{Discussion}

If the maximum mass of a white dwarf is $1.35 M_{\odot}$, a white dwarf companion would be expected for inclinations $i>51^{\circ}$. Ultramassive white dwarfs have been found to be either single or in close binary systems (Vennes \& Kawka 2008), as in the peculiar binary system RX J0648.0-4418 (Mereghetti et al. 2009). Constraining the secondary mass between 1.35 and $0.75 M_{\odot}$, the system is not expected to merge before 5.1 to $7.8 \mathrm{Gyr}$. In the low-mass range the merger would create an ultramassive white dwarf, while in the upper mass range the system would constitute a type 1a supernova candidate.

Figure 3 shows the locus of the ELM binary systems in a period versus ELM mass diagram. The data from Table 3 are compared to the mass-period relations from the WD+NS binary evolutionary scenarios of Nelson et al. (2004) and Tauris \& Savonije (1999). Similar trends are also predicted for the brighter star in binary systems with two white dwarf stars (Nelemans et al. 2001). Figure 3 also includes the long period WD+NS system PSR B0820+02d (Koester \& Reimers 2000). This particular system extends the apparent correlation toward higher mass $\left(0.60 \pm 0.08 M_{\odot}\right)$ and longer period $(1232.5 \mathrm{~d})$. Table 3 first provides the parameters of NLTT 11748, followed by the parameters of systems containing white dwarfs with a neutron star companion (NS+WD), and double white dwarf systems $(\mathrm{WD}+\mathrm{WD})$. Finally, we list the parameters of the possible progenitors of ELM white dwarf binary systems. Some 
Table 3. Confirmed binary systems containing a low-mass white dwarf.

\begin{tabular}{lccc}
\hline \hline Name & $P_{\text {orb }}$ (days) & $M_{\mathrm{WD}}\left(M_{\odot}\right)$ & Reference \\
\hline NLTT 11748 & 0.2351 & $0.167 \pm 0.005$ & 1,2 \\
\hline \multicolumn{4}{c}{ NS + WD } \\
PSR B1855+09 & 12.3272 & $0.258 \pm 0.020$ & 3 \\
PSR J0437-4715 & 5.7410 & $0.254 \pm 0.018$ & 4 \\
PSR J0218+4232 & 2.0288 & $0.275 \pm 0.105$ & 5,6 \\
PSR J1909-3744 & 1.5334 & $0.204 \pm 0.002$ & 7 \\
PSR J1911-5958A & 0.8371 & $0.175 \pm 0.010$ & 8,9 \\
PSR J1012+5307 & 0.6047 & $0.16 \pm 0.02$ & $10,11,12$ \\
PSR J1738+0333 & 0.3548 & $0.20 \pm 0.05$ & 13 \\
PSR J0751+1807 & 0.2631 & $0.191 \pm 0.015$ & 14 \\
\hline \multicolumn{4}{c}{ WD + WD } \\
LP400-22 & 1.010 & $0.190 \pm 0.004$ & 15 \\
SDSS J0822+2753 & 0.244 & $0.17 \pm 0.01$ & 16 \\
SDSS J1257+5428 & 0.1898 & $0.20 \pm 0.05$ & 17 \\
SDSS J0849+0445 & 0.079 & $0.17 \pm 0.01$ & 16 \\
SDSS J1436+5010 & 0.0458 & $0.23 \pm 0.01$ & 16 \\
SDSS J1053+5200 & 0.0426 & $0.22 \pm 0.01$ & 16 \\
\hline \multicolumn{4}{c}{ Progenitors } \\
HD 188112 0.6066 & $0.24 \pm 0.10$ & 18 \\
SDSS J1023+0038 & 0.1981 & $0.2 \pm 0.05$ & 19,20 \\
\hline
\end{tabular}

References. (1) This work; (2) Kawka \& Vennes (2009); (3) Kaspi et al. (1994); (4) Verbiest et al. (2008); (5) Navarro et al. (1995); (6) Bassa et al. (2003); (7) Jacoby et al. (2005); (8) Corongiu et al. (2006); (9) Bassa et al. (2006); (10) Webb et al. (2004); (11) van Kerkwijk et al. (1996); (12) Callanan et al. (1998); (13) Freire et al. (2008); (14) Nice et al. (2005); (15) Vennes et al. (2009); (16) Kilic et al. (2010); (17) Marsh et al. (2010); (18) Heber et al. (2003); (19) Archibald et al. (2009); (20) Wang et al. (2009).

scatter between the mass of the brighter star and the orbital period is clearly visible, but a correlation is still observed between the two parameters. The models of Tauris \& Savonije (1999) appear to better represent the observed trend than those of Nelson et al. (2004). The two outstanding points that are significantly off the predicted relationship of either models are the two systems with very short periods (SDSS J1053+5200, SDSS J1436+5010: Kilic et al. 2010; Mullally et al. 2010). Overall, WD+WD and WD+NS systems overlap in the diagram, so this trend alone does not allow one to distinguish between $\mathrm{WD}+\mathrm{WD}$ and WD+NS systems.

The ELM white dwarfs that are companions to neutron stars appear to be very faint $(V>20)$ with the brightest known ELM companion to a neutron star with an apparent magnitude of $V=19.6$ (PSR J1012+5307). The binary systems containing an ELM white dwarf and either a confirmed or probable white dwarf companion are significantly brighter, with apparent visual magnitudes ranging from 16.5 up to 19.3 , with NLTT 11748 the brightest of the sample. In terms of distances we find that the WD+WD sample lies within a range of $0.1-1.6 \mathrm{kpc}$ at an average distance of $0.75 \mathrm{kpc}$, while the WD+NS sample lies within a range of $0.15-4.4 \mathrm{kpc}$ at an average distance of $1.5 \mathrm{kpc}$. Located at a distance of $0.4 \mathrm{kpc}$ and being the brightest known ELM, NLTT 11748 fits well within the relatively closer and brighter class of WD+WD systems. Moreover, NLTT 11748 is not associated with a known radio source, therefore based on these considerations alone we may conclude that the companion to NLTT 11748 is another white dwarf with a mass above average.

Indeed, after this work was submitted for publication, Steinfadt et al. (2010) announced the discovery of primary and secondary eclipses in NLTT 11748 confirming the secondary star as a white dwarf. Their analysis confirms the ELM nature of the primary star and corroborates our analysis of the binary properties. Their orbital period measurement, based on eclipse timing, agrees with our spectroscopic period within error bars. Finally, our ephemeris allows us to identifiy the primary and secondary eclipses observed by Steinfadt et al. (2010) with the corresponding orbital conjunctions.

\section{Conclusions}

We find that the ELM white dwarf NLTT 11748 is part of a short-period binary system $(P=5.64 \mathrm{~h})$. Based on our orbital analysis alone, we find that the minimum mass of the companion is $0.75 M_{\odot}$. However, the companion has independently been identified as a faint white dwarf (Steinfadt et al. 2010), and assuming a mass of $0.75 M_{\odot}$, we constrained the companion temperature to $\leq 9600 \mathrm{~K}$ (DA) or $\leq 7200 \mathrm{~K}$ (DC). The kinematics of NLTT 11748 also place the system in the Galactic halo.

Acknowledgements. A.K. and S.V. are supported by GA AV grant numbers IAA301630901 and IAA300030908, respectively, and by GA ČR grant number P209/10/0967. We thank the referee for a prompt review and useful suggestions.

\section{References}

Agüeros, M. A., Camilo, F., Silvestri, N. M., et al. 2009a, ApJ, 697, 283 Agüeros, M. A., Heinke, C., Camilo, F., et al. 2009b, ApJ, 700, L123 Archibald, A. M., Stairs, I. H., Ransom, S. M., et al. 2009, Science, 324, 1411 Badenes, C., Mullally, F., Thompson, S. E., \& Lupton, R. H. 2009, ApJ, 707, 971

Bassa, C. G., van Kerkwijk, M. H., \& Kulkarni, S. R. 2003, A\&A, 403, 1067

Bassa, C. G., van Kerkwijk, M. H., Koester, D., \& Verbunt, F. 2006, A\&A, 456, 295

Benvenuto, O. G., \& Althaus, L. G. 1999, MNRAS, 303, 30

Callanan, P. J., Garnavich, P. M., \& Koester, D. 1998, MNRAS, 298, 207 Corongiu, A., Possenti, A., Lyne, A. G., et al. 2006, ApJ, 653, 1417

Freire, P. C. C., Jacoby, B. A., \& Bailes, M. 2008, AIP Conf. Proc., 983, 488

Heber, U., Edelmann, H., Lisker, T., \& Napiwotzki, R. 2003, A\&A, 411, L477

Henry, T. J., \& McCarthy, Jr., D. W. 1993, AJ, 106, 773

Jacoby, B. A., Hotan, A., Bailes, M., Ord, S., \& Kulkarni, S. R. 2005, ApJ, 629, L113

Kaspi, V. M., Taylor, J. H., \& Ryba, M. F. 1994, ApJ, 428, 713

Kawka, A., \& Vennes, S. 2009, A\&A, 506, L25

Kilic, M., Brown, W. R., Allende Prieto, C., et al. 2009, ApJ, 695, L92

Kilic, M., Brown, W. R., Allende Prieto, C., \& Kenyon, S. J. 2010, ApJ, 716, 122

Koester, D., \& Reimers, D. 2000, A\&A, 364, L66

Kulkarni, S. R., \& van Kerkwijk, M. H. 2010, ApJ, submitted [arXiv: 1003.2169]

Marsh, T. R., Gänsicke, B. T., Steeghs, D., et al. 2010, ApJL, submitted [arXiv: 1002 . 4677]

Mereghetti, S., Tiengo, A., Esposito, P., et al. 2009, Science, 325, 1222

Mullally, F., Badenes, C., Thomson, S. E., \& Lupton, R. 2010, ApJ, 707, L51

Navarro, J., de Bruyn, A. G., Frail, D. A., Kulkarni, S. R., \& Lyne, A. G. 1995, ApJ, 455, L55

Nelemans, G., Yungelson, L. R., Portegies Zwart, S. F., \& Verbunt, F. 2001, A\&A, 365, 491

Nelson, L. A., Dubeau, E., \& MacCannell, K. A. 2004, ApJ, 616, 1124

Nice, D. J., Splaver, E. M., Stairs, I. H., et al. 2005, ApJ, 634, 1242

Serenelli, A. M., Althaus, L. G., Rohrmann, R. D., \& Benvenuto, O. G. 2002, MNRAS, 337, 1091

Steinfadt, J. D. R., Kaplan, D. L., Shporer, A., Bildsten, L., \& Howell, S. B. 2010, ApJ, 716, L146

Tauris, T. M., \& Savonije, G. J. 1999, A\&A, 350, 928

van Kerkwijk, M. H., Bergeron, P., \& Kulkarni, S. R. 1996, ApJ, 467, L89

Vennes, S., \& Kawka, A. 2008, MNRAS, 389, 1367

Vennes, S., Kawka, A., Vaccaro, T. R., \& Silvestri, N. M. 2009, A\&A, 507, 1613

Verbiest, J. P. W., Bailes, M., van Straten, W., et al. 2008, ApJ, 679, 675

Wang, Z., Archibald, A. M., Thorstensen, J. R., et al. 2009, ApJ, 703, 2017

Webb, N. A., Olive, J.-F., Barret, D., et al. 2004, A\&A, 419, 269

Zhang, X., Chen, X., \& Han, Z. 2009, A\&A, 504, L13 